\title{
Enquête
}

Archives de la revue Enquête

4 | 1996

La ville des sciences sociales

\section{La ville-mémoire}

Quelques usages de La Mémoire collective de Maurice Halbwachs

City as memory. Some uses of Maurice Halbwachs' La Mémoire collective

\section{Sylvie Mazzella}

\section{(2) OpenEdition \\ Journals}

Édition électronique

URL : http://journals.openedition.org/enquete/883

DOI : 10.4000/enquete.883

ISSN : 1953-809X

Éditeur :

Cercom, Éditions Parenthèses

Édition imprimée

Date de publication : 1 novembre 1996

Pagination : 177-189

Référence électronique

Sylvie Mazzella, « La ville-mémoire », Enquête [En ligne], 4 | 1996, mis en ligne le 12 juillet 2013,

consulté le 30 avril 2019. URL : http://journals.openedition.org/enquete/883 ; DOI : 10.4000/

enquete.883

Ce document a été généré automatiquement le 30 avril 2019. 


\title{
La ville-mémoire
}

\author{
Quelques usages de La Mémoire collective de Maurice Halbwachs \\ City as memory. Some uses of Maurice Halbwachs' La Mémoire collective
}

\section{Sylvie Mazzella}

1 Maurice Halbwachs, en écrivant La Mémoire collective, avait la préoccupation de distinguer clairement le «métier de sociologue» du «métier d'historien ${ }^{1}$ ». Travailler sur la mémoire collective c'était, pour lui, écrire, contre l'histoire des historiens, une histoire locale et matérielle, urbaine, à hauteur des groupes. L'ambition de la mémoire collective est en effet de restituer une " histoire vivante ", concrète et multiple, dans les interstices de l'histoire. Halbwachs jette ainsi, presque à son insu, les bases d'une complexification du temps historique.

2 La multiplicité du social à partir de laquelle il pense la mémoire s'enracine fortement dans l'observation du milieu urbain : une des façons de comprendre cette invention de la mémoire collective est de la considérer comme un renouvellement de la notion de tradition, liée d'habitude à une pensée terrienne de l'histoire, en particulier à une histoire $\mathrm{du}$ « pays». La ville d'Halbwachs est un espace dynamique en constante transformation, défini comme un ensemble de territoires perçus, paradoxalement, par les habitants comme stables, où s'enracine la mémoire des groupes. On lui doit d'avoir su donner, à la suite de l'école de Chicago, une définition topographique du groupe social dans la ville et d'avoir lié la question de l'identité collective à l'appropriation locale du passé.

Depuis la fin des années quatre-vingt, un certain nombre de travaux sur la ville qui prennent en compte la dimension temporelle du groupe et du territoire, l'analyse des modes d'articulation entre configurations spatiales et temporalités sociales, trouvent dans les écrits de Maurice Halbwachs une source renouvelée d'inspiration. Il est dès lors nécessaire de revenir au texte, de préciser son contexte intellectuel, de définir les différentes pistes qu'il ouvre dès l'origine. Et ce, non pour en donner une version canonique, mais pour en discerner aujourd'hui les différentes valeurs d'usage et leurs enjeux. Ce rappel de la référence halbwachsienne veut aussi signifier que le nécessaire 
effort d'adaptation et de réappropriation de la notion ne peut être efficace que dans la mesure où le texte de La Mémoire collective continue de faire résistance.

\section{Le temps de la mémoire} épistémologique autonome; il veut en particulier redéfinir sociologiquement les catégories d'espace et de temps. Durkheim, en particulier des Formes élémentaires de la vie religieuse (1912), et de Bergson, singulièrement de Matière et Mémoire (1897). Bergson, Durkheim et Halbwachs se retrouvent pour critiquer le caractère apriorique, abstrait et universel des notions philosophiques de temps et d'espace. Bergson demande, dans la première page de Matière et Mémoire, d'oublier, pour commencer, les discussions philosophiques, pour raisonner à partir de la perception des choses ${ }^{2}$. Durkheim s'attaque lui directement à Kant et attribue une origine historique et sociale aux catégories universelles de temps et d'espace ${ }^{3}$. Quant à Halbwachs, il ne dissimule pas son refus du «temps abstrait des philosophes ", à l'opposé du «temps réel». C'est dans cet esprit critique, propre au contexte philosophique de l'époque, qu'il redéfinit les notions de temps et d'espace, en infléchissant l'un par l'autre l'objectivisme de Durkheim et le subjectivisme de Bergson.

\section{Critique du temps subjectif}

Le modèle temporel travaillé par Halbwachs est celui de toute la tradition métaphysique. Le temps est tendu entre deux pôles : le temps cosmique, universel, objectif et mesurable, dans lequel nous passons, et le temps vécu, individuel et subjectif, celui qui passe en nous. La critique de ce modèle se fait en deux étapes. En premier lieu, il socialise le temps individuel, le seul réel selon Bergson. Il attribue ensuite à ce temps social vécu toutes les caractéristiques du temps individuel bergsonien. Comme toute fonction humaine, la mémoire a pour cadre la société, c'est dans et pour la société que l'individu se souvient. Le premier geste de La Mémoire collective est, en effet, de socialiser le temps subjectif : notre perception du temps est immergée dans le social. «On admettra qu'il se crée une sorte de milieu artificiel, extérieur à toutes pensées personnelles, mais qui les enveloppe, un temps et un espace collectifs et une histoire collective. C'est dans de tels cadres que les pensées des individus se rejoindraient ${ }^{4}$. »

7 Ce temps social endosse toutes les caractéristiques de la durée subjective bergsonienne, dont la principale est la multiplicité, "qualitative », " hétérogène », " altérable ». Plus encore qu'une expérience psychologique première, le temps est l'essence variable des choses. En fait, l'opposition d'Halbwachs à Bergson n'est pas celle du collectif contre l'individuel, mais de la primauté de l'un sur l'autre : c'est le temps collectif qui permet les durées individuelles et non l'inverse ${ }^{5}$.

\section{Critique du temps social}

Pour Durkheim, le temps social impersonnel est, au-dessus des individus, une « véritable institution ». C'est le rôle qu'il attribue au temps calendaire comme forme objective de 
l'activité sociale ${ }^{6}$. Pour Halbwachs, tout au contraire, le calendrier est une « construction abstraite » qui ne peut pas être considérée comme le temps social puisque des individus appartenant à des sociétés différentes s'y réfèrent. Que le calendrier ait une origine religieuse historique, voilà qui explique, pour Durkheim, son caractère social ; voilà qui confirme son aspect non collectif pour Halbwachs: il s'agit d'une "survivance », d'une "vieille idée ", d'une tradition éclatée, qui a pris aujourd'hui des significations très différentes suivant les groupes. On voit que l'analyse diffère sur un point central : la question du temps historique. Chez Durkheim, les temps sociaux particuliers sont subsumés par une institution temporelle continue, cumulatrice et homogène. Au contraire pour Halbwachs, à la suite de Bergson, le temps constitue «l'essence variable des choses ", à l'opposé de l'espace, chiffre de la stabilité. Il est multiple parce que perçu différemment par une multitude de groupes sociaux. Les groupes ne cumulent pas leur expérience au fil du temps; ils s'adaptent, résistent, et meurent. La mémoire collective est l'instance qui permet au groupe de résister malgré le temps qui passe, de se stabiliser dans la durée, « une spatialisation du temps » selon la formule bergsonienne. Halbwachs ne considère pas l'histoire comme dimension positive de l'humanité. Le temps de la discipline historique est reconstruit, abstrait, non sociologique : l'histoire se place hors des groupes, au-dessus d'eux. Il distingue ainsi la « reconstruction » des événements par l'histoire de la «reconstitution» par la mémoire collective. L'auteur, qui vise alors principalement l'histoire institutionnelle nationale, rejette le temps historique du côté de l'universel, du "temps abstrait » et la mémoire collective du côté du temps vécu, du « temps réel ». Il n'ignore certes pas qu'au même moment s'élabore une histoire nouvelle qui défend une conception de la société proche de la sienne - il appartient au comité de rédaction des Annales d'histoire économique et sociale dès leur fondation. Cela n'empêche pas qu'en fin de compte, dans son aspiration, "l'histoire est une» et qu'il existe fondamentalement, pour lui, deux disciplines fondées sur deux modes de temporalités différentes ${ }^{7}$.

9 À travers le champ de la mémoire collective Halbwachs défend une stricte sociologie du présent, présent « entendu comme s'étendant sur une certaine durée, celle qui intéresse la société d'aujourd'hui ", c'est-à-dire une durée homogène et stable de la vie d'un groupe, telle que la contient la mémoires.

\section{La mémoire élargie}

10 Lorsqu'il conclut son étude de la mémoire collective chrétienne, Halbwachs s'attache surtout à répondre à Durkheim sur l'origine sociale du phénomène religieux. On voit comment sa définition de la mémoire collective s'est singulièrement élargie dans une étude proprement historique: "La mémoire collective chrétienne adapte à chaque époque ses souvenirs [...] aux exigences contemporaines du christianisme, [...] à ses aspirations. Seulement dans cet effort d'adaptation, les hommes se heurtent à la résistance des choses [...] aux vestiges matériels des croyances anciennes [...]. Mais, quelque époque qu'on envisage, l'attention se porte non vers l'origine, les événements premiers qui sont peut-être à l'origine de tout ce développement, mais vers les groupes [...]. Dans la physionomie [...] des lieux saints [...] c'est bien l'essentiel du phénomène religieux dont ces pierres dressées et conservées par les générations successives des hommes permettent de retrouver les traces. Traces non pas d'un être individuel, humain ou surnaturel, mais des groupes. ${ }^{9}$ " 
11 La Topographie légendaire peut être considérée comme un texte durkheimien dans la mesure où il retrace la formation d'un fait social, l'histoire de la localisation des événements néotestamentaires. Le phénomène religieux y est étudié comme un fait social dominant qui organise l'espace. L'espace de la Topographie est d'ailleurs moins territorial que symbolique. C'est celui de la chrétienté, mieux du christianisme, un espace quasi total, sur lequel une tradition en mouvement - la mémoire collective - trace et retrace des itinéraires et des hauts lieux, « la physionomie des lieux saints ». C'est ce qu'indique J.-C. Passeron quand il écrit que la démarche d'Halbwachs s'inscrit « dans une sociologie durkheimienne de l'exploration de l'amont des conduites. » Il en est ainsi «tant que le groupe de transformation [...] peut se définir et se décrire comme topographique ». La Topographie est un texte durkheimien dans la mesure où il retrace la " genèse laborieuse » d'un fait social, dans la mesure surtout où la mémoire collective semble comprise comme déterminant social, comme institution durkheimienne ${ }^{10}$. Mais Halbwachs ignore l'origine de cette mémoire, ou plus exactement il l'ignore comme raison causale, comme «forme primitive ». La mémoire n'est pas, comme pour Durkheim, l'outil qui permet la remontée à l'origine. Pour Durkheim, comme l'écrit G. Namer, « la mémoire de la religion doit être renvoyée à une sociologie du progrès ${ }^{11}$ ". Pour Halbwachs au contraire, elle ne fait que rendre compte des aspirations du moment et de la résistance des choses. En un sens, phénomène religieux et mémoire n'ont pas d'origine, encore moins une origine qui en serait la forme simplifiée, "élémentaire ». En étudiant l'itinéraire d'une mémoire collective d'un "groupe de transformation », depuis l'espace territorial vécu jusqu'à l'espace symbolique parsemé de « lieux de mémoire », l'auteur décrit les changements de la mémoire d'un groupe qui se délocalise, se généralise, s'universalise en peuple, incarné dans l'Église, et devient une « communauté élargie », possédant une " mémoire collective élargie $^{12} »$.

Ce que décrit Halbwachs c'est la formation d'une mémoire collective institutionnelle. Dans La Topographie légendaire, l'analyse passe du groupe menacé au groupe dominant, d'un espace territorial à un espace symbolique, fictif. Le groupe social n'affirme plus sa conscience de soi en s'appuyant sur l'espace matériel, il affirme son autorité par son emprise sur un espace total qu'il marque en des lieux choisis. Le groupe dessine son itinéraire sur l'espace géométrique et de fait devient topographe. L'auteur finit par opposer deux mémoires : celle affective, floue et fluctuante, des "groupes locaux » (les groupes territorialisés tels que les décrivait La Mémoire collective) qu'il nomme «les traditions locales", et celle réfléchie, cohérente, dogmatique, de la communauté chrétienne en son ensemble, qui détermine l'espace de la tradition ${ }^{13}$. L'opposition de ces deux mémoires, comme leur coexistence, présage de la diversité de l'usage actuel de la notion de mémoire collective.

\section{La mémoire des historiens}

13 L'histoire, en son sens institutionnel, en même temps qu'elle se pose en gardienne des mémoires collectives, a toujours eu pour souci de les unifier, de réduire la multiplicité des groupes à une vision univoque du passé. L'histoire est une rectification de la mémoire, voire "sa néantisation ». " Au cœur de l'histoire travaille un criticisme destructeur de mémoire ", écrit P. Nora ${ }^{14}$. L'historiographie française en particulier, depuis le XVI ${ }^{\mathrm{e}}$ siècle, et plus particulièrement à la fin $d u \mathrm{XIX}^{\mathrm{e}}$, s'est présentée comme un hymne collectif à la gloire de la nation. La contestation de cette mémoire unitaire s'est produite à deux 
niveaux, épistémologique et social ${ }^{15}$. D'une part, par la recherche d'une nouvelle histoire attentive aux variations de perspectives, à la multiplicité des rythmes temporels et des approches problématiques. D'autre part, par l'analyse de multiples mémoires de groupes sociaux minoritaires ou dominés (classe ouvrière, minorités régionales ou culturelles etc.) dont l'ambition est d'accéder à la reconnaissance institutionnelle. «Il y avait autrefois une histoire nationale et des mémoires particulières; il y a aujourd'hui une mémoire nationale mais dont l'unité est faite d'une revendication [...] en permanente démultiplication et recherche de cohésion ${ }^{16}$. Dans ce contexte, on comprend que des historiens aient trouvé dans la mémoire collective d'Halbwachs un outil de travail approprié. Les fables de la mémoire de L. Valensi, étude sur quatre siècles des variations littéraires du récit d'une bataille chez les vainqueurs et les vaincus, retient explicitement d'Halbwachs les "variations de la mémoire" telles qu'elles s'observent dans La Topographie légendaire, les transformations du souvenir, les "affabulations", accordées aux aspirations des groupes et du moment ${ }^{17}$. Les lieux de mémoire, réunis par P. Nora, sont une tentative, " entre histoire et mémoire ", pour saisir la multiplicité historiographique et sociale des symboles nationaux. «À la solidarité du passé et de l'avenir » (la nation comme héritage et la nation comme projet) s'est substituée « la solidarité du présent et de la mémoire ». Mais pour P. Nora la mémoire est moins l'outil d'analyse d'une société en crise perpétuelle, en transformation constante, que le signe d'une crise de l'histoire ${ }^{18}$.

Les historiens ont donc fini par reprendre un concept qui avait été élaboré contre eux en transférant à l'étude de la nation un concept créé pour l'étude de la société urbaine.

\section{Les temporalités urbaines}

C'est un autre courant de recherches qui a ramené Halbwachs sur son terrain urbain. Dans un cadre interdisciplinaire, la plupart des auteurs qui l'invoquent, depuis la fin des années quatre-vingt, s'intéressent aux temporalités urbaines ${ }^{19}$. Qu'ils soient attentifs à la morphologie urbaine, aux groupes et à leurs territoires, ou aux flux migratoires, ils empruntent à l'auteur de La Mémoire collective l'idée de décalage temporel entre le groupe et son espace, du fait même de son inscription dans un territoire en transformation. C'est à ce titre que la notion de résistance forgée par Halbwachs sert de référence aujourd'hui. Il est bon d'en rappeler le cadre.

\section{La notion de résistance}

16 M. Halbwachs énonce l'idée que le fondement de la mémoire collective se trouve dans sa projection dans des espaces concrets, que les pierres sont indissociables des formes de mémorisation et des représentations qui agissent sur la ville. Notons que tous les groupements sociaux se constituent sur une base spatiale, même ceux qui «en apparence » «font abstraction » du « lieu qu'ils occupent ${ }^{20}$ ». Un groupe lié à son espace le «transforme à son image en même temps qu'il s'y plie et s'adapte ». Certes l'espace est apparemment stable mais quatre types de processus viennent le former.

17 En premier lieu, le temps initial de l'installation et de l'adaptation. C'est le temps fondateur de "l'emplacement » où se crée «l'adhérence du groupe à sa place ", où prend forme l'image stable du lieu ${ }^{21}$. 
18 À l'opposé logique et chronologique, se situe la forme de la disparition du groupe : étape finale d'une inadaptation du groupe social, de la persistance de formes socio-spatiales dépassées: «Il en est ainsi [...] de tous les modes d'activités un peu anciens, qui n'ont guère leur place dans les cités modernes. Ils [...] disparaîtraient sans doute s'ils ne s'attachaient obstinément aux lieux qui leur étaient autrefois réservés ». Ils disparaissent finalement pour ne laisser que "vestiges matériels", «îlots archaïques", reliquats de leur obstination.

La troisième forme est la seule à bénéficier chez Halbwachs du terme de transformation. Elle emprunte à $\mathrm{G}$. Simmel sa conception de la modernité. Si les groupes ont besoin d'un temps d'adaptation, et sont voués à terme à l'inadaptation, c'est qu'il existe un temps qui les dépasse, les enveloppe; le temps inéluctable de la modernisation, «des grands courants ", le temps événementiel des accidents et des catastrophes, des incendies et des invasions, des démolitions et des reconstructions: le temps de l'histoire. Les grandes transformations sont une sorte de fond sonore qui n'affecte que peu, ou rarement, la vie des groupes : «Les habitants se trouvent porter une attention très inégale à ce que nous appelons l'aspect matériel de la cité, mais le plus grand nombre sans doute serait plus sensible à la disparition de telle rue, de tel bâtiment, de telle maison, qu'aux événements nationaux, religieux, politiques les plus graves ${ }^{22}$.»

De la rencontre entre ce temps historique de la modernité, des accidents, et le temps immobile des choses matérielles naît la quatrième forme de la transformation chez Halbwachs, la plus novatrice et la plus féconde : la résistance du groupe aux forces de changement. En effet, "si les villes se transforment au cours de leur histoire », "les habitudes locales résistent aux forces qui tendent à les transformer» et qui "les troublent et les déconcertent ». Les groupes laissent des traces, des survivances, de leur existence révolue. Ce sont ces traces qui expliquent la permanence de formes urbaines anciennes.

21 Ainsi s'explique que la ville ne soit pas qu'une simple partition cartographique de territoires collectifs, mais une superposition de plans en constant réajustement. Halbwachs construit ainsi une épaisseur historique de la ville. Il jette les bases d'une histoire sociale urbaine.

\section{Contemporanéité urbaine}

22 C'est cette leçon du texte d'Halbwachs que privilégie le groupe de recherches sur «les temps des villes ", autour de M. Roncayolo et B. Lepetit, ou les chercheurs du CRESAL sur la trace urbaine ${ }^{23}$. Prolongeant l'analyse d'Halbwachs puis celle de l'historien J.-C. Perrot sur la permanence des villes ${ }^{24}$, B. Lepetit parle de régression à l'infini des processus d'adaptation et de résistance des groupes qui fait de la ville le lieu d'un processus permanent de réélaboration. $\mathrm{M}$. Roncayolo a insisté sur le fait que les villes sont moins des idéaux-types - «ville baroque, haussmannienne, industrielle»- que des " composés ». La ville est un constant décalage entre espace réel et espace projeté. Il a pris le cas marseillais comme un laboratoire des ruptures et déplacements de valeurs qui sont l'expression et la modalité du changement urbain. "Ces besoins et ces anticipations [...] s'expriment à travers des schémas et des contradictions situés dans l'espace, alors même qu'ils contribuent à en assurer la modification ou le dépassement. Ainsi Halbwachs a-t-il défini $[. .$.$] un temps urbain { }^{25}$.» 

de « remplois », d'une « mise au présent » des formes anciennes : « La configuration de la ville, note B. Lepetit, résulte de l'enchaînement de l'ensemble des configurations précédentes. » On passe d'une sociologie des groupes urbains à une histoire des modalités d'appropriation sociale de la forme urbaine. Les travaux de B. Lepetit font le pont entre l'Halbwachs de l'école de Chicago et celui de la mémoire, et se réclament à la fois de la sociologie urbaine de La Mémoire collective et de l'approche historique de La Topographie légendaire. Lepetit s'appuie pour cela chez Halbwachs, non sur l'identité pérenne d'un groupe à travers l'histoire - la communauté chrétienne-, mais sur l'exemple de l'évolution de la morphologie d'une cité, celle de Jérusalem, reconstruite par les Croisés à partir de l'image biblique qu'ils en avaient. Au-delà de cet exemple, l'efficacité de la mémoire collective tient dans sa capacité à lier spatial et temporel, à problématiser la ville comme objet d'histoire, quitte à devoir repenser l'une et l'autre ${ }^{26}$. chercheurs du CRESAL qui en dressent une typologie. La coexistence d'éléments matériels d'époques différentes à l'intérieur d'un quartier témoigne de différentes «logiques de présence ». Certains éléments sont "actifs », d'autres "passifs ». Cette anthropologie urbaine tente de concilier la recherche de documents - une archéologie urbaine - et l'examen des principes de perception et d'interprétation des éléments matériels de la ville. On a pu montrer, par exemple, dans un travail précédent, comment la figure du chantier qui caractérise depuis bientôt trente ans le quartier Belsunce dans le centre ancien de Marseille, avec palissades, grues et terrains vagues, était à la fois revendiquée par l'institution politique qui y voit la visibilisation de ses efforts d'éradication d'une population stigmatisée, et par certaines familles maghrébines, anciennement implantées, qui y puisent une croyance en la revalorisation de leur territoire.

Sur le même terrain, A. Tarrius, étudiant la logique de territorialisation des groupes de migrants, définit le territoire urbain «non comme une forme spatiale » mais comme un temps social ${ }^{27}$. Enfonçant le coin des divergences entre La Mémoire collective et $\mathrm{La}$ Topographie légendaire là où $\mathrm{B}$. Lepetit tente de les réduire, il affirme : « Nous ne pouvons nous satisfaire seulement d'une vision historique des villes. M. Halbwachs après s'être épuisé à relever, des années durant, les morphologies urbaines découvrait l'essentialité de la mémoire collective, puis, dans les derniers mois de son existence, la capacité des groupes sociaux à remodeler par la désignation les formes de leur environnement pour

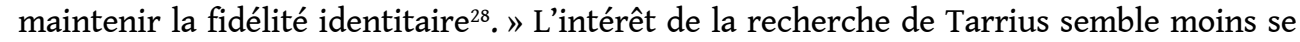
situer dans la qualité de l'espace, « territoire circulatoire » qui associe l'espace migratoire et celui de l'ordre marchand, que dans le renversement d'optique qui consiste à traiter un groupe, les migrants maghrébins, jusque-là considéré dominé dans l'espace urbain, comme dominant dans l'espace transnational. C'est en ce sens qu'il a raison de se réclamer de l'Halbwachs de La Topographie, dont on a vu qu'elle décrivait, en fin de compte, la mémoire institutionnelle d'un groupe hégémonique, qui marque de son emprise un territoire fictif, virtuel.

Le rapport entre histoire nationale et sociologie urbaine que tentait d'établir de manière simplifiée M. Halbwachs, est ainsi reposé de manière intra-nationale par une histoire de la morphologie urbaine et extra-nationale par une histoire des mouvements migratoires. A. Tarrius pourrait reprendre l'affirmation de B. Lepetit selon laquelle «la question clef de la citoyenneté est celle de l'articulation entre les lieux et les communautés, entre lieux 
réels et lieux fictifs ${ }^{29}$ ». L'espace fictif c'est l'espace du réseau que le groupe emporte avec lui dans ses migrations et qu'il reconstitue là où il se pose.

L'exemple marseillais est de ce point de vue significatif. Ce qui est nommé « quartier arabe ", au cœur de la ville, est considéré par les uns comme le ghetto d'une communauté minoritaire dominée, par les autres comme lieu de passage et objet d'effacement de la mémoire par les politiques urbaines (É. Temime), ou encore comme nœud urbain d'un réseau marchand international, un territoire virtuel, déplaçable (A. Tarrius). Une attention plus précise aux discours de la territorialisation des habitants immigrés, permet de repérer non seulement leur attachement aux transformations du lieu jugées valorisantes quoique destinées le plus souvent à les invisibiliser, mais aussi une revendication $d u$ passé bourgeois du quartier (architecture bourgeoise du $\mathrm{xvIII}^{\mathrm{e}}$ d'appartements réhabilités qu'ils occupent, relais des postes du xvIII ${ }^{e}$ classé monument historique abritant la mosquée principale de Marseille). Le thème de l'intégration est particulièrement révélateur des qualités heuristiques de la mémoire collective en ce qu'il pose abruptement à la fois les questions de l'adaptation d'un groupe urbain à son lieu, de l'articulation entre territoire réel et territoire fictif, et de l'appartenance nationale, la nation comme sol des ancêtres ou comme mémoire partagée (R. Park).

On entrevoit, d'une manière plus générale, comment une démarche anthropologique de recueil de la mémoire collective urbaine, du discours de l'appropriation du passé local vécu et non vécu, permet de réinterpréter l'histoire d'un lieu en prenant en compte le récit des différents groupes sociaux, menacés ou dominants, qui l'occupent.

La plupart des auteurs français se référant à $M$. Halbwachs insistent sur la nécessité actuelle de partir des atomes élémentaires, des groupes, des « trajectoires individuelles et sociétales ». C'est en effet dans « les interstices de l'histoire » que M. Halbwachs créait sa sociologie de la mémoire. À nouveau, aujourd'hui, «le lieu du débat est dans les interstices spatiaux et sociaux" (A. Tarrius). C'est dans la crise des grands modèles explicatifs que la problématique de la mémoire réapparaît, crise de l'histoire morale et politique autrefois, crise de l'histoire socio-économique aujourd'hui. L'appel à la mémoire est suivant les auteurs, le signe d'une crise provisoire de l'identité politique nationale (un moment-mémoire), l'outil approprié de la crise permanente des villes ou, pour ceux plus optimistes qui croient en la sortie de la crise, une des clés de compréhension des temporalités sociales multiples.

Dans un certain sens, au-delà des questions disciplinaires et de l'organisation institutionnelle du champ des connaissances, l'enjeu de la mémoire collective reste le même que du temps de $\mathrm{M}$. Halbwachs: réaffirmer l'opposition entre temporalité historique et temporalité sociologique. Et ce, non pour établir la supériorité de l'une sur l'autre, mais pour éclaircir une confrontation estompée par une pratique interdisciplinaire. Il s'agit moins d'opposer deux temps - quoique se remarquent les difficultés d'une histoire du présent ou d'une sociologie du passé - que deux temporalités. Une qui privilégie le lien envers les hommes du passé et prend appui sur un espace terrien où s'enracinent l'origine et la dette envers les morts d'ici - les ancêtres -, une autre qui privilégie le lien entre les hommes d'aujourd'hui et prend appui sur un espace en transformation - la ville -, où s'exacerbe l'obligation du présent, jusque dans l'appropriation du passé, pour saisir le processus contradictoire de l'enracinement urbain. 


\section{NOTES}

1. M. Halbwachs, La Mémoire collective [1950], Paris, Presses universitaires de France, 1968, rassemble des notes écrites dans les années quarante. Ce projet suit donc Les cadres sociaux de la mémoire, Paris, F. Alcan, 1925, et La Topographie légendaire des Évangiles en Terre Sainte. Étude de mémoire collective [1941], Paris, Presses universitaires de France, 1972, consacré à la mémoire collective de la communauté chrétienne.

2. «On verra que l'écueil de tout idéalisme est là dans ce passage de l'ordre qui nous apparaît dans la perception à l'ordre qui nous réussit dans la science - ou, s'il s'agit de l'idéalisme kantien, dans le passage de la sensibilité à l'entendement ", note H. Bergson, Matière et Mémoire [1897], Paris, Presses universitaires de France, 1993, p. 255.

3. Sur le contexte intellectuel et social où se développe la sociologie durkheimienne, cf. J.C. Chamboredon, «Émile Durkheim : le social, objet de science. Du moral au politique ? », Critique, 445-446, 1984, p. 461-497.

4. M. Halbwachs, La Mémoire collective, op. cit., p. 45.

5. Ibid., « Critique du subjectivisme bergsonien », p. 87-92.

6. « Un calendrier exprime le rythme et l'activité collective en même temps qu'il a pour fonction d'en assurer la régularité [...]. Ce n'est pas mon temps qui est ainsi organisé ; c'est le temps tel qu'il est objectivement pensé par tous les hommes d'une même civilisation ", É. Durkheim, Les formes élémentaires de la vie religieuse [1912], Paris, Presses universitaires de France, 1968, p. 14-15.

7. Dans le compte rendu qu'il donne, dès 1925 , des Cadres sociaux de la mémoire, M. Bloch exige une meilleure historisation de la notion de mémoire collective: "Une partie au moins des phénomènes que nous désignons ainsi sont tout simplement des faits de transmission de génération en génération. » Il suggère aussi le thème d'une histoire " des erreurs de la mémoire collective » qui permettent de réinterpréter régulièrement le récit biblique, thème repris onze ans après par Halbwachs dans la Topographie légendaire des évangiles. Bloch réclame ce que Halbwachs déplore: l'histoire comme rectification de la mémoire. («Mémoire collective, tradition et coutume », Revue de Synthèse historique, 118-120,1925, p. 73-83).

8. Conformément à la pensée de Bergson, la durée halbwachsienne se définit moins par la succession que par la coexistence du passé et du présent. « C'est l'utilité de la situation présente, note ainsi G. Deleuze, qui crée le mouvement, le moteur, qui sélectionne le niveau de passé et l'actualise pour qu'il présente sa face utile. » (Le Bergsonisme, Paris, Presses universitaires de France, 1966, p. 58-69.)

9. M. Halbwachs, La Topographie légendaire, op. cit., p. 164.

10. J.-C. Passeron, Le raisonnement sociologique. L'espace non-poppérien du raisonnement naturel, Paris, Nathan, 1991, p. 77.

11. G. Namer, postface de la réédition des Cadres sociaux de la mémoire, Paris, Albin Michel, 1994, p. 303.

12. «Il y eut une période où la communauté chrétienne n'était pas reconnue officiellement, où elle était combattue et persécutée [...]. L'image des lieux saints est demeurée dans la mémoire des groupes chrétiens, et surtout d'une Église élargie qui gagnait en étendue et en consistance. » ( $L a$ Topographie légendaire, op. cit., p. 127-128.)

13. G. Namer, analysant le texte des Cadres sociaux de la mémoire, oppose lui aussi deux mémoires, mais sensiblement différentes, « d'un côté une mémoire fidèle à la tradition qu'elle regarde et de l'autre une raison attentive tant aux valeurs du monde présent qu'aux déterminations de ce présent » (op. cit.). 
14. «Tel est le sérieux de l'histoire : elle se propose de raconter les civilisations du passé et non de sauver la mémoire des individus ", écrit aussi P. Veyne. La position morale de l'historien estelle impliquée par le devoir de mémoire, la dette envers les morts où P. Ricœur situe la raison historienne, ou bien excentrée par un scepticisme critique, par «l'exotisme» des faits historiques dont parle P. Veyne?

15. Cf. les remarques de N.Wachtel, «History and Memory. Introduction", History and Anthropology, II, 1986, p. 207-224. Remarquons ici que c'est avec l'introduction, dans les années soixante-dix, des « archives orales ", que les historiens en France se mettent à lire l'Halbwachs de La Topographie légendaire et de La Mémoire collective.

16. P. Nora, Les lieux de mémoire, t. III, Les France, 3, De l'archive à l'emblème, Paris, Gallimard, 1992, p. 1009.

17. Pour L. Valensi, le modèle halbwachsien est littéraire non seulement parce que le matériau est textuel - récits de voyages médiévaux pour Halbwachs, chroniques ou poèmes pour Valensi -, mais parce qu'il s'agit de comprendre les représentations collectives de chaque époque comme une réécriture de l'histoire. Les exercices de style de Queneau sont un des modèles des Fables de la mémoire, Paris, Seuil, 1992.

18. Sur l'actualité du débat, cf. L. Valensi, "Histoire nationale, histoire monumentale ", et F. Hartog, « Temps et histoire », Annales HSS, L, 1995, p. 1271-1277, 1219-1236 ; G. Noiriel, Sur la « crise » de l'histoire, Paris, Belin, 1996.

19. M. Roncayolo souligne la "nécessaire interdisciplinarité » des recherches sur la ville, une ville aux temporalités multiples, "dans ses développements jamais achevés ": la ville comme crise et provisoire et permanente, comme histoire sans fin, Le Courrier du CNRS, 81, 1994, p. 7.

20. M. Halbwachs, «L'espace fictif », in La Mémoire collective, op. cit., p. 140-145.

21. Ibid., p. 139.

22. Ibid., p. 134.

23. B. Lepetit, Les temps des villes. Bilan d'un programme de recherche, Paris, Plan urbain, 1993. D. Colson, J. Nizey et J. Roux, Un quartier dans le temps, Paris, Plan urbain, 1993. Ces auteurs s'inspirent d'Halbwachs et aussi de la réflexion de P. Ricœur sur la trace comme effet-signe. Entre l'empreinte laissée ici et maintenant et l'événement révolu, la trace, synthèse de deux temporalités contradictoires, condense les problèmes de la construction historique. P. Ricœur, Temps et récit, t. III, Paris, Seuil, 1985, p. 217-227.

24. J.-C. Perrot, Genèse d'une ville moderne : Caen au XVIII siècle, Paris-La Haye, Mouton, 1975 ; cf. en particulier p. 553: «Les maisons anciennes et les ateliers, plus encore les édifices publics, les voies et les places fossilisent, pour de longues périodes, des usages, des activités mourantes dont il faut s'accommoder malgré le présent. La trame urbaine possède ainsi une faible viscosité et si quelques quartiers en leur ensemble dérivent d'un façonnement homogène et chronologiquement plat, le centre des villes est presque toujours un conservatoire temporel. Les traces, sur le sol, de l'activité et de l'appropriation humaines y sont peut-être plus durables qu'à la campagne où l'on connaît la lourde permanence des champs de nos pays d'Occident. Première habitude écologique des disparus, la ville façonne la nature des vivants. »

25. M. Roncayolo, Les grammaires d'une ville. Essai sur la genèse des structures urbaines à Marseille, Paris, Éd. de l'EHESS, 1996, p. 58.

26. B. Lepetit, «Le présent de l'histoire », in B. Lepetit, ed., Les formes de l'expérience. Une autre histoire sociale, Paris, Albin Michel, 1995, p. 273-298.

27. A. Tarrius, Arabes de France dans l'économie mondiale souterraine, La Tour d'Aigues, Éd. de l'Aube, 1995. Dans la même perspective, cf. le dernier ouvrage de M. Roncayolo, Marseille, les territoires du temps, Paris, Éditions locales de France, 1996.

28. A. Tarrius, Les fourmis d'Europe. Migrants riches, migrants pauvres et nouvelles villes internationales, Paris, L'Harmattan, 1992.

29. B. Lepetit, Les temps des villes..., op. cit. 


\section{RÉSUMÉS}

Depuis la fin des années quatre-vingt, La Mémoire collective constitue une importante source d'inspiration pour l'analyse des modes d'articulation entre configurations spatiales et temporalités urbaines. Les différentes pistes que ce texte ouvre expliquent en partie les points de vue divergents actuels, en particulier entre sociologues et historiens. Dans la ville d'Halbwachs, espace dynamique en constante transformation, la mémoire des groupes sociaux, définie comme réappropriation du passé accordée aux aspirations du présent, constitue leur mode privilégié de territorialisation. À l'opposé d'une vision terrienne de l'histoire, la mémoire collective se présente comme un des outils privilégiés de l'enquête urbaine, d'une construction de l'histoire de la ville.

Since the end of the 1980's, La Mémoire collective constitutes an important source of inspiration for the analysis of modes of articulation between spatial configurations and urban temporalities. The different paths that this text opens partly explain the current divergent points of view, particularly between sociologists and historians. In Halbwachs' city, a dynamic space in continuous change, the memory of social groups, defined as the reappropriation of the past reconciled to the aspirations of the present, constitute their priviliged way of territorialisation. As opposed to a terrestrial vision of history, collective memory appears as one of the privileged tools of an urban inquiry, of the construction of the city's history.

\section{AUTEUR}

\section{SYLVIE MAZZELLA}

Sylvie Mazzella (EHESS-CNRS) travaille en sociologie urbaine sur Marseille, principalement sur la question de l'intégration sociale dans les dynamiques urbaines. Elle a publié récemment « Le quartier Belsunce à Marseille : Les familles maghrébines sur les traces de la ville bourgeoise », in Annales de la Recherche urbaine, «Patrimoine et modernité », n 72, 1996. 